\title{
Topical patches as treatments for the management of patient musculoskeletal and neuropathic pain
}

\author{
Simona Mirel ${ }^{1}$, Liora Colobățiu ${ }^{1}$, Mihaela Mirel$^{2}$, Sorina Pop ${ }^{2}$ \\ 1. Faculty of Pharmacy, "Iuliu Hatieganu" University of Medicine and Pharmacy Cluj-Napoca, Romania \\ 2. Faculty of Medicine, "Iuliu Hatieganu" University of Medicine and Pharmacy Cluj-Napoca, Romania \\ smirel@umfcluj.ro
}

\begin{abstract}
The variety and multiple dimensions of pain (acute/chronic, mild/moderate/severe, nociceptive/neuropathic) requires different pharmacologic and non-pharmacologic treatments in certain patients. A lot of topical formulation, from various therapeutic classes have been proposed in order to decrease systemic exposure and to reduce the risks of adverse events. Topical as well as transdermal drug delivery systems are proposed as medicated plasters with: anesthetics (lidocaine), analgesic or nonsteroidal anti-inflamatory drugs (NSAIDs), alone or co-formulated. Capsaicin, salicylates, menthol and camphor represent the counterirritant class of topical analgesics used as patch active compounds. These compounds produce their analgesic effect by activating and desensitizing epidermal nociceptors. The most used topical treatment in order to decrease pain is the application of cold and heat patches - by acting directly on the affected tissue. In many cases there is a limited number of studies providing insufficient information to clinicians in order to evaluate the benefits of these products. This paper reviews the use and efficacy of available self-adhesing occlusive medicated plaster (pain patches) that might represent an alternative option for the management of patient pain, specially in the case of musculoskeletal and neuropathic disorders.
\end{abstract}

Key words: musculoskeletal and neuropathic pain, patch, medicated plaster, efficacy

\section{Background:}

A variety of health products are available for the management of chronic painful conditions, including both systemic and topical analgesics, as well as several alternative methods. Due to the fact that oral pharmaceuticals for pain have significant side effects, the use of topical analgesics is recommended for their good safety profile, low risk for systemic and patient compliance [1].

Many health products have been use as topical analgesics and claiming efficacy in a variety of painful conditions, particularly for musculoskeletal, arthritic or neuropathic pain. There are several pharmaceutical preparations available on the market, in a plaster/patch format, with analgesic and antiinflammatory action, which provides pain relief. Compared with semi-solid dosage forms, the plaster formulation offers the advantage of easy handling, once a day applying and uniform releasing of active substance, with favorable risk-benefit ration.

\section{- Medicated-patches}

The medicated-patches containing local anesthetic (lidocaine) [2-4] or antiinflammatory (NSAIDs) are used to manage patients with select pain condition (acute and chronic). A topical patch containing diclofenac epolamine, available in more than 40 countries worldwide, was approved for use in Europe since 1993 (2007 in USA), and the available postmarketing surveillance data offers good safety data [5-7]. The local treatment of soft-tissue rheumatism with flurbiprofen (40mg) patch versus oral therapy with diclofenac sodium provided both efficacy and good tolerability [8]. The clinical trials in patients with non-articular rheumatism and traumatic painful soft tissue injuries showed that the patches containing ketoprofen (100 $\mathrm{mg}$ as the active principle) was significantly effective at reducing pain 
after 7 days' treatment [9]. The efficacy of a betamethasone valerate medicated plaster was reported in improving pain and functional disability in patients with arthritis and osteoarthritis [10].

According with different regulatory process these product are commercially available as OTC drugs (over-the-counter) [11-16] (tab. 1) or medical devices. Most products are not reviewed by European Medicines Agency (EMA) or U.S. Food and Drug Administration (FDA), however they could be marketed if they comply with the policies and regulations applicable.

Tab.1. Example of analgesics and anesthetics medicated patches for musculoskeletal and neuropathic pain registered as drugs (EMA and FDA)

\begin{tabular}{|l|l|l|l|}
\hline Drugs & $\begin{array}{l}\text { Trade } \\
\text { name }\end{array}$ & Dose & Posology \\
\hline Lidocaine & Lidoderm & $\begin{array}{l}700 \mathrm{mg} / \\
140 \mathrm{~cm}^{2}\end{array}$ & $\begin{array}{l}\text { only once } \\
\text { for up to } \\
12 \mathrm{~h}\end{array}$ \\
\hline & Versatis & & \\
& Flector & $140 \mathrm{~cm}^{2}$ & \\
Diclofenac & $140 \mathrm{mg}$ & up to 12h \\
& Voltarol & $140 \mathrm{~cm}^{2}$ & \\
\hline $\begin{array}{l}\text { Capsaicin } \\
\text { Qutenza }\end{array}$ & $\begin{array}{l}179 \mathrm{mg} / \\
280 \mathrm{~cm}^{2}\end{array}$ & $\begin{array}{l}\text { single } \\
60 \text { min } \\
\text { applic. up } \\
\text { to 12h }\end{array}$ \\
\hline $\begin{array}{l}\text { Menthol } \\
\text { (M) } \\
\text { /Methyl } \\
\text { salicylate } \\
\text { (MS) }\end{array}$ & Salonpas & $\begin{array}{l}31.5 \mathrm{mg}, \\
\text { (M); } \\
105 \mathrm{mg} \\
\text { (MS)/ } \\
70 \mathrm{~cm}^{2}\end{array}$ & $\begin{array}{l}\text { up to } \\
8-12 \mathrm{~h}\end{array}$ \\
\hline
\end{tabular}

The available comercially analgesics patches contain: capsaicin, menthol, methyl salicylate, camphor or different herbal extracts. Many formulations assembled combinations of these ingredients.

Several topical formulations are commercially available on pharmaceutical market as medical devices (risk class III, according to EU classification rule no. 13 - devices incorporating a substance that can be considered to be a medicinal product by pharmacological means) [17] and this is the aim of this paper. The products are, usually, the counterirritants, warming plasters and are indicated for temporary treatment of minor pain due to backache, sprains, bruises, arthritis, or diabetic neuropathy. The term patch/plasters is limited to products that apply topical analgesic active compounds to the skin by means of an adhesive matrix and not refer to transdermal delivery systems.

The self-treatment with these semiocclusive patches for pain, must be limited and should be referred to the primary health care provider, in order to have an adequate medical care and treatment [18].

\section{- Patch formulations containing Capsaicin}

The most used are capsaicin patch, marketed as a topical preparation indicated for relief of local pain. It is considered that the adhesive plasters containing capsaicin (capsicum oleoresin or capsicum extract) are intended for heat-treatment, local analgesia.

Capsaicin (a naturally alkaloid derived from genus Capsicum - hot chili peppers) is intended for heat-treatment, indicated to treat rheumatic, muscular and neuralgic pains. Due to the fact that topical capsaicin is well absorbed and rapidly through the skin, the products are available in a variety of over-the-counter (OTC) formulations (creams, gels, patches) and concentrations (from $0.025 \%$ to $0.15 \%$ ) [19, 20].

Its pharmacological actions are explained through selectivity of capsaicin for the TRPV1 receptor and the expression of TRPV1 in nociceptive sensory nerves. Thus, capsaicin affects the TRPV1 receptor (involved in sensing heat, warmth and pain), which transmits pain and itch sensations from the peripheral to the central nervous system. The TRPV1 receptor stimulated by capsaicin, releases sensory neuropeptides and blocks axonal transport in sensory neurons, resulting the diminution of pain perception (19). There is no evidence that 
topical capsaicin works through a transdermal systemic delivery into tissues other than the skin. Capsaicin is a lipophilic compound, non-water-soluble, presenting therefore a limited potential for transdermal delivery in humans. Even if the capsaicin is absorbed systemically, the duration of exposure is very short [20].

There are several clinical studies proving the efficacy of plaster capsicum formulations as suitable for the treatment of musculoskeletal and neuropathic pain, with or without inflammatory components. According with European Guidelines for the management of chronic non-specific low back pain, Capsicum plasters can be considered, for short-term, symptomatic pain relief in chronic low back pain [21].

Different studies used different pain scales, and the secondary efficacy measures were tests of mobility, a disability index and global assessments by physicians and patients. Keitel, Frericck and colab, in two double-blind, randomised parallel-group study (154 patients - using Capsicum Pain Plaster $11 \mathrm{mg}$, respectively 320 patients using Capsaicin Heat Patch $4.8 \mathrm{mg}$ ) suggest the use of both capsicum plaster as an alternative in the treatment of non-specific back pain. The superiority of the treatment with capsicum plaster compared to placebo was considered clinically relevant and statistically significant (responders rate in the capsicum group of more than $60 \%$ for capsicum group [22, 23].

Different formulation proposed (with different capsaicin low-concentration) allows a once daily application instead of 3 to 4 applications of a cream. The advantage of the capsaicin-patch is that this drug is poorly absorbed transdermally across human skin and shows only a few local adverse effects (site reactions such as pain and erythema).

Capsaicin in the form of $8 \%$ dermal patch is approved - in the EU and USA - as drugs for neuropathic pain associated with postherpetic neuralgia and is available by prescription. A 60 minutes application on patients with neuropathic pain produce effective pain relief for up to 3 months after administration [15, 24]. The effect of a highconcentration capsaicin patch on healthy volunteers were evaluated by epidermal nerve fiber (ENF) density and quantitative sensory testing (QST) 24 weeks after capsaicin exposure. The results predict the long-term safety of the patch applications [25].

\section{- Patch formulations containing methyl salicylate, camfor, menthol}

The occlusive patch formulations containing methyl salicylate, camfor, menthol - alone or in different combination are also topical analgesic, recommended for the treatment of mild to moderate pain. The available patches could be single-entity or combination formulations: different compounds on different concentrations (menthol $0,4 \%$ to $6 \%$; methyl salicylate 0,8 to $6,2 \%$, camfor $0,5-1,2 \%$ ).

Although these are extensively used, there is little information concerning the pharmacokinetics of those compounds following dermal application. Also, published efficacy and safety data regarding these patches use are limited.

Methyl salicylate action is due to an inhibitory effect on prostaglandin biosynthesis. Camphor has analgesic and anti-inflammatory properties and has been shown to activate heat-sensitive transient receptor potential (TRP) vanilloid, acts on TRPV1 and TRPV3 receptors. Levomenthol has been reported to have analgesic effects, including anesthesic action, acts on TRPM8 and TRPA1 receptors, secondary to the activation of endogenous opioid receptors $[16,26]$.

Topical rubefacients cause irritation and reddening of the skin, due to increased blood flow. They are used in the treatment of pain in various musculoskeletal conditions

Some adverse events reported may be suggestive of some degree of systemic exposure. Martin and colab. report the dermal absorption of camphor, menthol and methyl salicylate in humans using a selective gas-chromatographic method. The plasma concentrations of each compounds suggests a 
low systemic exposure to these potentially toxic compounds, even when a large number of patches are applied for a long time [27].

Regarding efficacy and safety data, Higashi Y and colab. report that a single, 8hour application of a patch containing methyl salicylate and l-menthol provided significant relief of pain associated with mild to moderate muscle strain in these adult patients compared with placebo patches [28]. But their use could be associate with application - site reactions (erythema, dryness or burning) The US FDA, in a Drug Safety Communication, has alerted the public that certain OTC topical muscle and joint pain relievers - especially the patches containing menthol as the single active ingredient at $3 \%$ or more and methyl salicylate combinations above $10 \%$ - may cause burns [29].

\section{- Patch formulations containing traditional herbal extracts}

There are many products commercially available in the market (also on Romanian pharmaceutical market) from certified Chinese manufacturers, well-known as pain relief patches. The main ingredients used in anti-rheumatic herbal patches are Boswellic acid and Curcumin. The producers propose different combinations of traditional formula, based on Chinese herbal medicine. Common ingredients include capsicum, menthol and a mixture of natural plant herbs., and Radix Aconiti, Myrrha, Olibanum, Rhizoma Ligusticum, Radix Clematisdis [30]. The active ingredients penetration into the affected area .and provides temporary pain relief (6-8 h).

These patches might offer some improvement of pain symptoms, but there are available only several comprehensive study for their effectiveness and safety.

\section{- Cold and heat patches}

On the pharmaceutical market, there are also many others patch formulations, intended to be used for mild to moderate muscular pain. The most used topical treatment in order to decrease pain is the application of cutaneous patch - cold and heat - by acting directly on the affected tissue (without reaching the target tissue through systemic distribution). Heat therapy patches function as counterirritants agents, by creating an alternate sensation on the skin. There is a lack of studies regarding the actions and the efficacy of these products and this could be the aim of future work studies.

\section{Conclusion}

Acute and chronic pain condition require a selection of therapeutical arsenal for long-term use. Topical effect could be achieved using nonsteroidal antiinflammatory, anesthetics or analgesics drugs and/or other ingredients with counterirritant effects. There are several health products commercially available as medicated and warming plasters/ patches, which can be considered for short-term treatment of musculoskeletal and neuropathic pain. are intended to be used for mild to moderate pain, for no longer than 7 days. Generally, their efficacy claims are based only on the improvement of pain symptoms in common usage, and there are not many controlled trials concerning the use of these products. 


\section{References}

1. Jorge LL, Feres C, Teles V. Topical preparations for pain relief: efficacy and patient adherence. J Pain Res. 2011; 4: 11-24.

2. Argoff CE. New analgesics for neuropathic pain: The lidocaine patch. Clin J Pain 2000;16:S62-6.

3. Devers A, Galer BS. Topical lidocaine patch relieves a variety of neuropathic pain conditions: Anopen-label study. Clin J Pain 2000;16:205-8.

4. Comer AM, Lamb HM. Lidocaine patch 5\%. Drugs 2000;59:245-9.

5. Li C, Frangione V, Rovati S, Zheng Q. Diclofenac epolamine medicated plaster in the treatment of minor soft tissue injuries: a multicenter randomized controlled trial. Curr Med Res Opin. 2013 29(9):1137-46.

6. McCarberg BH1, Argoff CE. Topical diclofenac epolamine patch $1.3 \%$ for treatment of acute pain caused by soft tissue injury. Int J Clin Pract. 2010; 64(11):1546-53.

7. Kuehl KS1. Review of the efficacy and tolerability of the diclofenac epolamine topical patch $1.3 \%$ in patients with acute pain due to soft tissue injuries. Clin Ther. 2010; 32(6):1001-14.

8. Martens M1. Efficacy and tolerability of a topical NSAID patch (local action transcutaneous flurbiprofen) and oral diclofenac in the treatment of soft-tissue rheumatism. Clin Rheumatol. 1997;16(1):25-31.

9. Mazières B. Topical ketoprofen patch. Drugs R D. 2005; 6(6):337-44.

10. Frizziero A, Causero A, Bernasconi B et al. Efficacy of betamethasone valerate medicated plaster on painful chronic elbow tendinopathy: a doubleblind, randomized, placebo-controlled trial. Muscles Ligaments Tendons J. 2016; 6(1): 131-139.

11. Lidoderm ${ }^{\circledR}$-Lidocaine Patch 5\%, http://www.accessdata.fda.gov/drugsatfda_docs/label/ 2005/020612s007lbl.pdf

12. Versatis ${ }^{\circledR} \quad-\quad 5 \% \quad$ Medicated Plaster, https://www.medicines.org.uk/emc/medicine/19291

13. Flector Patch ${ }^{\circledR}-1.3 \%$, diclofenac epolamine patch

http://www.accessdata.fda.gov/drugsatfda_docs/label/ 2011/021234s005lbl.pdf

14. Voltarol ${ }^{\circledR}$ Gel Patch - $1 \%$ diclofenac epolamine medicated plaster, https://www.medicines.org.uk/emc/medicine/16903 15. Qutenza $^{\circledR}$ - $179 \mathrm{mg}$ cutaneous patch, http://www.ema.europa.eu/docs/en_GB/document_lib rary/EPAR_-

_Product_Information/human/000909/WC500040453. pdf

16. Salonpas Pain Relief Patch $^{\circledR}$, http://www.accessdata.fda.gov/drugsatfda_docs/nda/2 008/022029TOC.cfm

17. Guidance document - Classification of Medical Devices-EDDEV 2.4/1 rev.9, available at http://ec.europa.eu/consumers/sectors/medicaldevices/files/meddev/
18. Wright E. Musculoskeletal Injuries and Disorders. In: Berardi RR, Kroon LA, McDermott JH, et al, eds. Handbook of Nonprescription Drugs. 15th ed. Washington, DC: American Pharmacists Association; 2006:111-129

19. O'Neill J, Brock C, Olesen AE, Andresen T, Nilsson M, Dickenson AH. Unravelling the mystery of capsaicin: a tool to understand and treat pain, Pharmacol Rev. 2012; 64(4):939-71.

20. Sharma SK, Vij AS, Sharma M. Mechanisms and clinical uses of capsaicin. Eur J Pharmacol. 2013; 720(1-3):55-62.

21. Airaksinen O., Brox J. I., Cedraschi C. et al, European guidelines for the management of chronic nonspecific low back pain. Eur Spine J. 2006. ; 15 (Suppl. 2): S192-S300.

22. Frerick H, Keitel W, Kuhn U, Schmidt S, Bredehorst A, Kuhlmann M. Topical treatment of chronic low back pain with a capsicum plaster. Pain. 2003;106(1-2):59-64.

23. Keitel W, Frerick H, Kuhn U, Schmidt U, Kuhlmann M, Bredehorst A. Capsicum pain plaster in chronic nonspecific low back pain. ArzneimForsch/Drug Res 2001; 51(11):896-903.

24. Anand P, Bley K: Topical Capsaicin for Pain Management: Therapeutic Potential and Mechanisms of Action of the New High-concentration Capsaicin 8\% Patch. Brit J Anaesthes 2011; 107(4):490-502

25. Kennedy WR, Vanhove GF, Lu SP et al. A randomized, controlled, open-label study of the longterm effects of NGX-4010, a high-concentration capsaicin patch, on epidermal nerve fiber density and sensory function in healthy volunteers. J Pain. 2010 (6):579-87.

26. Kotaka T, Kimura S, Kashiwayanagi M, Iwamoto J. Camphor induces cold and warm sensations with increases in skin and muscle blood flow in human. Biol Pharm Bull. 2014; 37(12):1913-8.

27. Martin D, Valdez J, Boren J, Mayersohn M. Dermal absorption of camphor, menthol, and methyl salicylate in humans. J Clin Pharmacol. 2004; 44(10):1151-7.

28. Higashi Y, Kiuchi T, Furuta K. Efficacy and safety profile of a topical methyl salicylate and menthol patch in adult patients with mild to moderate muscle strain: a randomized, double-blind, parallelgroup, placebo-controlled, multicenter study. Journal of Clinical Therapeutics. 2010;32(1):34-43

29. Over-The-Counter Topical Muscle and Joint Pain Relievers: Drug Safety Communication - Rare Cases of Serious Burns, available: http://www.fda.gov/Safety/MedWatch/SafetyInformat ion/SafetyAlertsforHumanMedicalProducts/ucm3193 53.htm

30. Wang X, Wei S, Liu T et al. Effectiveness, Medication Patterns, and Adverse Events of Traditional Chinese Herbal Patches for Osteoarthritis: A Systematic Review. Evidence-based Complementary and Alternative Medicine. 2014;(4):343176. 